\title{
QUANTITATION AND REGIONAL DISTRIBUTION OF SWEAT GLANDS IN MAN ${ }^{1}$
}

\author{
By WALTER C. RANDALL \\ (From the Department of Physiology, Saint Louis University School of Medicine, St. Louis)
}

(Received for publication May 13, 1946)

The distribution of sweating in normal and pathological conditions has been the subject of many investigations, and many methods for qualitative and quantitative measurement of the amount of sensible and insensible perspiration have been described. A survey of available literature has revealed fewer methods for quantitation of the number of functionally active sweat glands, and none which combine the technical simplicity and accuracy of the method herein described.

Of the so-called colorimetric methods, Minor's classical starch-iodine test (1) is the most commonly used. This method is not useful in quantitative studies however, and is subject to clinical objection, because of the necessity of application of a starch paste with a resultant black stain in a positive test. Roth (2) reported a clinical test for sweating in which active secretion is marked by a color change in cobalt chloride on the skin, and Silverman and Powell (3) report a colorimetric test involving a reaction between ferric chloride and tannic acid in the presence of active sweating. Gurney and Bunnell (4) described a method in which paper is floated over silver nitrate solution and exposed to ultraviolet light ; sweating is indicated by small brown spots of reduced silver nitrate. Direct microscopic examination of active sweat pores is reported by Kuno (5), and by Lobitz and Osterberg (6), and Buley (10).

\section{METHOD}

The method described below involves the reaction of starch and iodine, but eliminates the necessity of applying a coating of starch powder over the skin. A dilute solution of iodine ( 2 to 3 per cent in 95 per cent alcohol) is painted over the area and allowed to dry. (For quantitative purposes we use a blank rubber stamp, $1 \mathrm{sq} . \mathrm{cm}$. in area, with which the iodine can be stamped on the area to be studied, thus providing a convenient and constant area in which to count the active glands.) A blank piece of ordinary bond paper is then pressed lightly over the

1 Preliminary presentation before the American Physiological Society at Atlantic City, March 14, 1946. area for 20 seconds. Although any starch-containing paper may be suitable, we have found that the smooth hard finish of number 13 Voucher Bond contains sufficient starch, yet prevents excessive diffusion, and produces very acceptable and reproducible records. As the paper is held in place over the area, water secreted from the sweat pores places the starch and iodine in solution thereby producing definite blue-black spots on the test paper. The size of the spots is a qualitative indication of the amount of secretion from each active sweat pore, i.e., the larger the spot, the greater the amount of sweat secretion from that particular sweat pore. If no sweating is present, no spot will appear on the test paper. If sweating is marked, it may be necessary to repaint the area after several such records have been made. Although these records have not proved reliably permanent for long periods of time, they produce clear and sharp points for immediate analysis, and remain clear for a period of several weeks when stored in a dark place. Permanent records are obtained by photographing the test papers.

The number of active glands is counted under the dissection microscope, or from an enlarged or projected photograph of the test paper. (For qualitative studies on sweating, microscopic examination is not necessary, because the sweat spots are clearly discernible on visual inspection.) With this technique we are able to study the general pattern of sweating on any part of the body, count the number of active glands in any given area, study changing patterns of activity, gain information concerning the nervous control of sweating, and determine directly the effects of physiological, pathological, or neurosurgical procedures involving the sudomotor mechanism.

\section{EXPERIMENTAL RESULTS}

Figure 1 illustrates the usefulness of the procedure in checking the effectiveness of operations involving the sympathetic supply to the extremeties. In this patient, the left upper extremity was partially sympathectomized, and it was desirable to know how effectively the operation had reduced sweating. Records 2 days after the operation showed a great reduction in the number of active sweat glands, but not complete abolition of sweating. There were a few active glands on all the terminal finger pads and upon thenar and hypothenar eminences, thus indicating a few remaining intact pathways to the sweat glands via both the ulnar 


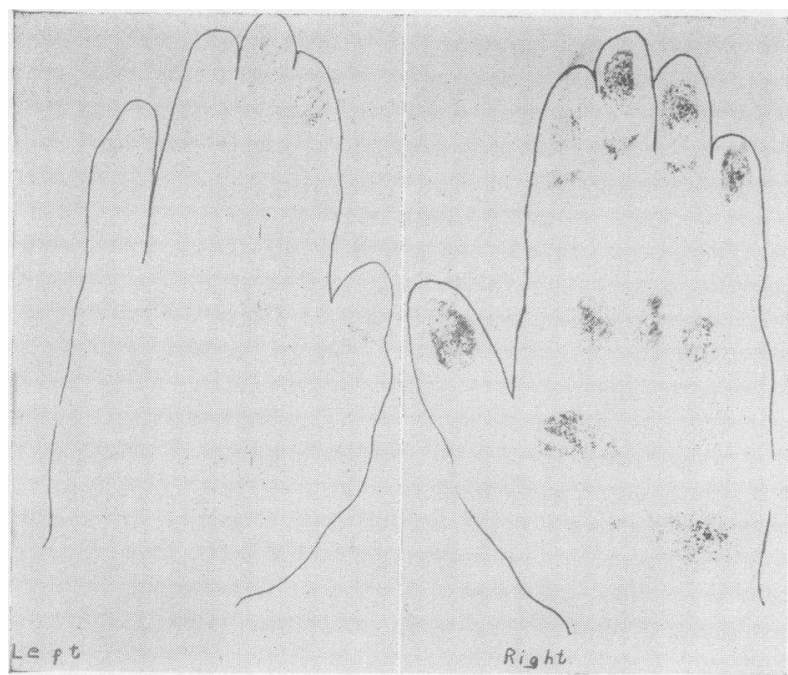

Fig. 1. Simultaneous Records of Sweating on the Normal Right Hand, and Partially Sympathectomized Left Hand, Palms of a Patient 11 Weeks After Operation

Iodine was applied to the terminal finger pads, to the pads covering the metacarpelphalangeal junction, and to the thenar and hypothenar eminences, and sweating in these areas considered representative of the palmar surfaces.

and median nerves. A relatively greater number of glands on the 1 st and 2 nd finger pads indicated a predominance of intact innervation via the median nerve. Records taken at suitable intervals thereafter confirmed this distribution (Figure 1).

\section{Determination of maximum number of functional sweat glands}

Experience has shown considerable variation in functional activity of the sweat glands in a given area. It therefore seemed desirable to establish certain reproducible conditions under which comparative studies might be made. Such studies could be accomplished under conditions of minimal, basal, or maximal activity. Of these 3 physiologically constant conditions, maximal activity proved most applicable. Since Dale and Feldberg (7) demonstrated chemical transmission of impulses to glandular cells, it has been accepted generally that the sweat glands are functionally activated through the mediation of acetyl choline. If this is true, maximal activity of functional sweat glands in a given area should be attained by introducing adequate amounts of acetyl choline into the dermal layers of the skin. Since acetyl choline is effective for but a short time in the body, it is more satisfactory to use a drug having similar physiologic properties, but which is more stable in the body. Acetyl-beta-methylcholine (mecholyl, 0.1 per cent $)^{2}$ was used for this purpose. In order to insure relatively even distribution over a large area, the drug was introduced by iontophoresis ( 5 to 10 minutes at 4.5 to 5.0 milliamperes) into an area about 40 to $50 \mathrm{~mm}$. square. Results obtained by this procedure were compared with results obtained by stimulation with locally applied radiant heat, hot tub bath, and with spontaneously occurring sweating in a warm room. The radiant heat was supplied by 100 watt lamp bulbs in reflectors placed 65 to $90 \mathrm{~mm}$. from the test area. The heating was intense enough to produce erythema, "felt hot" to the subject, and produced a skin temperature in the area of 40 to $43^{\circ} \mathrm{C}$. The hot bath experiments were carried out with the subject seated in hot water (40 to $44^{\circ} \mathrm{C}$.) which came to a level just below the umbilicus. The arms were slightly elevated above the water level.

Table I shows the comparative results of such stimulations as measured on the extensor surface of the forearm. In all instances the number of glands active following mecholyl (Figure 2) and during the hot tub bath, show close correspondence, and presumably represent maximal activity in this area. The number of glands activated by

2 Mecholyl (acetyl-beta-methylcholine chloride) furnished through the courtesy of Merck and Co.

TABLE I

Maximum number of functional sweat glands (number of glands per sq. cm.) on the extensor surface of the forearm during, or immediately following, different kinds of stimulation

\begin{tabular}{l|c|c|c|c}
\hline \hline Subject & $\begin{array}{c}\text { Spontaneous } \\
\text { peaks in } \\
\text { warm room } \\
(24 \text { to 31 }\end{array}$ & $\begin{array}{c}\text { Radiant heat } \\
\left(42 \text { to } 43^{\circ} \mathrm{C}\right)\end{array}$ & $\begin{array}{c}\text { Hot tub bath } \\
\left(40 \text { to } 44^{\circ} \mathrm{C}\right)\end{array}$ & Mecholyl \\
\cline { 2 - 3 } V R & 108 & 59 & 250 & $230+$ \\
& 132 & 200 & & \\
Ra & 98 & 89 & 238 & 252 \\
& 93 & 230 & 224 & \\
& 144 & & 240 & \\
Fo & 100 & & & \\
& 20 & 220 & $212-$ & 245 \\
& 100 & 200 & & \\
\hline
\end{tabular}




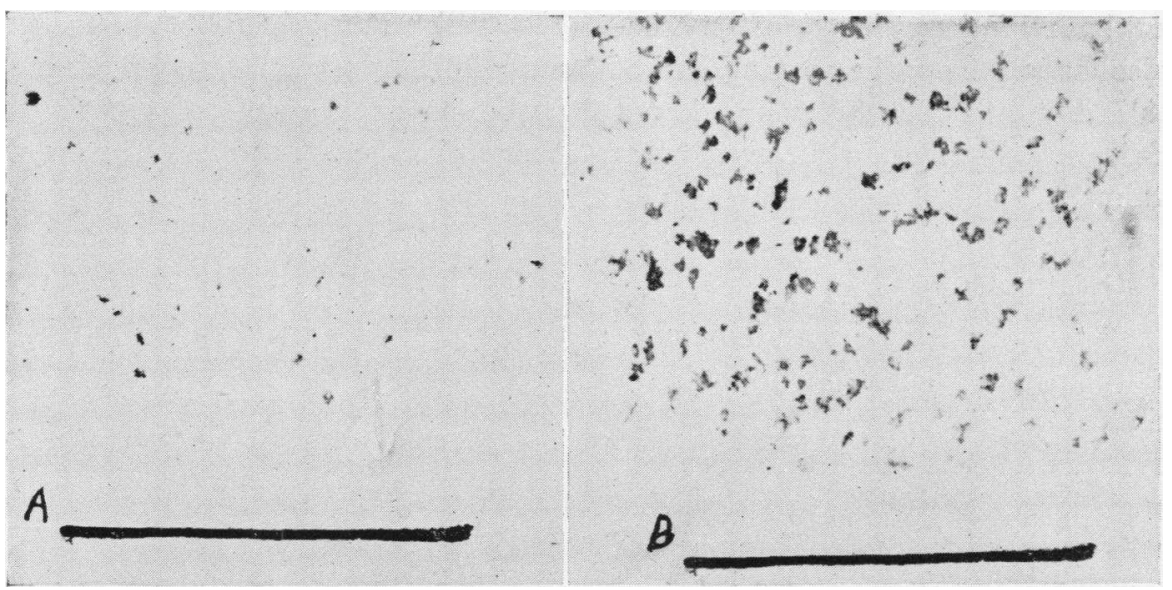

Fig. 2. Records Taken (A) Before and (B) After Mecholyl Iontophoresis on the Same Area of the Extensor Surface of the Forearm

The larger sweat spots in $B$ represent increased output, and in some instances the sweat from 2 or more pores which has run together. The calibration line represents $1 \mathrm{~cm}$. on the skin surface.

radiant heat depended upon the intensity of heat and extent of forearm heated, but in general this was not as effective a sudorific stimulus as the mecholyl or hot bath. This may be explained, possibly, by direct action of the drug in the one instance (mecholyl) and by the greater degree of summation, or a rise in blood temperature, in the other (hot bath experiments). The intensity of the stimulating temperature was designed to provide approximately comparable stimulating temperatures in both series of heat experiments. In all instances, the number of glands activated spontaneously in the warm room was least. These experiments have not been carried out at temperatures strictly comparable to those temperatures reached during the extreme heat of summer. Short exposures in a room temperature of $35^{\circ} \mathrm{C}$. however, did not activate more glands than indicated in Table I.

From these experiments it becomes apparent that relatively extreme thermal stimulation is required to activate simultaneously all of the functional sweat glands in a given area. Thermal stimulation followed by histological examination of the same area confirms this observation, as pointed out by Kuno (5) who quotes the work of Ogata. This worker counted the appearance of sweat droplets, followed by histological sectioning of the same tissues. He described glands which seemed morphologically well developed, but which did not appear to be functionally active under thermal stimulation. Of the "active glands," Ogata found variation from 125 to about 200 glands per sq. $\mathrm{cm}$. on comparable areas. These figures compare favorably with those contained in Table I, although we are inclined to believe there are more individuals showing the higher figure. Since we find that relatively extreme environmental temperatures are required to activate as many glands as direct chemical stimulation, it seems possible that Ogata's "inactive glands" represent glands which simply did not respond to the thermal stimulation employed. Others suggest the possibility that such inactive glands may represent glands which have been functional in infancy, but which have lost functional ability in later life. It is possible that the comparatively small discrepancies may have explanation in racial and climatic factors.

It should be pointed out briefly, that maximum activity denotes more than the participation of all of the functional glands in a given area, since it may also include an increased output by the individual glands. This is demonstrated in Figure 2, in which the size of many individual spots indicates increased output of sweat per unit period of time. Experience has shown that an increase in number of glands is not necessarily accompanied by increased output per gland, and the total volume of sweat produced by a given skin area may in- 
volve either or both of these functional mechanisms. It is also evident from the varying size of the sweat spots in Figure 2 that the glands do not participate equally in output of sweat, even when stimulated maximally.

\section{Distribution of sweat glands}

In extending studies of the maximum number of functional sweat glands to other parts of the body, cholinergic stimulation (mecholyl, 0.1 per cent solution) was used in small areas as described above. Although large individual variations become apparent as one compares the number of active glands on the same surfaces in different subjects, there are usually similar directional changes when one compares areas of high and low numerical counts on different subjects.

TABLE II

Distribution of functional sweat glands on different body surfaces

\begin{tabular}{l|r|r|r|r|r}
\hline \hline \multirow{2}{*}{ Area } & \multicolumn{4}{|c}{ Subject } \\
\cline { 2 - 5 } & Ra & Slo & Cla & She & Eng \\
\hline & \multicolumn{3}{|c|}{ (functional pores per sq.cm.) } \\
\begin{tabular}{l|r|r|r|r} 
Forearm, extensor \\
$\quad$ surface
\end{tabular} & 252 & 180 & 195 & & 225 \\
$\begin{array}{l}\text { Upper arm, over biceps } \\
\text { Dorsum of hand }\end{array}$ & 220 & 140 & & & 170 \\
Trunk & 410 & 260 & 480 & 416 & 320 \\
$\quad \begin{array}{l}\text { anterior chest } \\
\text { scapular region of back }\end{array}$ & 175 & 93 & & & 184 \\
Leg (over gastrocnemius) & 116 & 85 & 44 & 29 & 17 \\
Thenar eminence & 368 & 264 & 440 & 146 & 130 \\
Face & & & & & \\
$\quad$ forehead & 212 & $37(?)$ & & & 122 \\
zygomatic & 14 & 1 & 7 & 65 & 20 \\
buccal & 34 & 9 & & & 6 \\
\hline
\end{tabular}

Mecholyl was used in stimulating activity in all regions except thenar eminence in Subject Ra. Question in Subject Slo indicates probable inhibition of sweating by mecholyl iontophoresis.

Of the areas studied in Table II, the palmar surfaces and the dorsum of the hand are sites of greatest concentration of sweat glands. Although counts on the normally sweating finger pad may show as many as 500 or more active glands per sq. cm., stimulation with mecholyl in these areas has not consistently produced maximum counts. In fact, contrasting results have been observed following mecholyl iontophoreses, and inhibition of sweating in this area has been demonstrated when dilutions identical to those producing maximum sweating in other areas is used. It is commonly observed, on the other hand, that close to the maximum number of glands may be activated spontaneously, or as a result of emotional excitement. These divergent responses to mecholyl, combined with the lack of sweating during thermal stimulation (8), emphasizes the variant characteristics of the palmar sweat glands. Reference to Figure 3 (segment I) demonstrates the definite pattern of distribution of sweat pores which marks the palmar surfaces, and particularly the terminal finger pads. The sweat pores are small and somewhat irregularly spaced along the epidermal ridges. Although no pores have been observed between the ridges, sweat sometimes spills over into the shallow depressions and thereby produce elongated prints between rows of spots. Sweat output by individual glands on the palmar surface is relatively large, hence the exposure in Figure 3 (segment Hand I) was reduced to prevent coalescence of the sweat spots.

Whereas the hypothenar eminence shows marked ridges and definite rows of sweat spots on the test papers, the thenar eminence is less definitely ridged and marked by folds and creases which interrupt the spacing of the sweat pores (Figure 3, segment $\mathrm{H}$ ). The pores are less densely concentrated than on the finger pads, but are still numerous when compared with non-palmar surfaces.

The dorsum of the hand is marked by a surprisingly large number of sweat pores per sq. $\mathrm{cm}$., particularly when compared with forearm surfaces. The output per gland per unit of time on the dorsum appears to be significantly less than on forearm or upper arm surfaces, as indicated by simultaneous records taken from points $3 \mathrm{~cm}$. below (segment $\mathrm{C}$ ) and $3 \mathrm{~cm}$. above (segment $\mathrm{B}$ ) the wrist. Thus, although the sweat pores are considerably more numerous on the dorsum of the hand than on other surfaces of the arm, the total output of sweat per unit area may not be significantly different.

Measurements on the trunk were made from the anterior wall of the chest and from the back over the scapular region. In all subjects many more functional glands were observed on the chest wall, with a pattern of distribution quite similar to that observed on the upper arm. Considering the large surface area represented by the trunk and extremities, the number of glands and output 


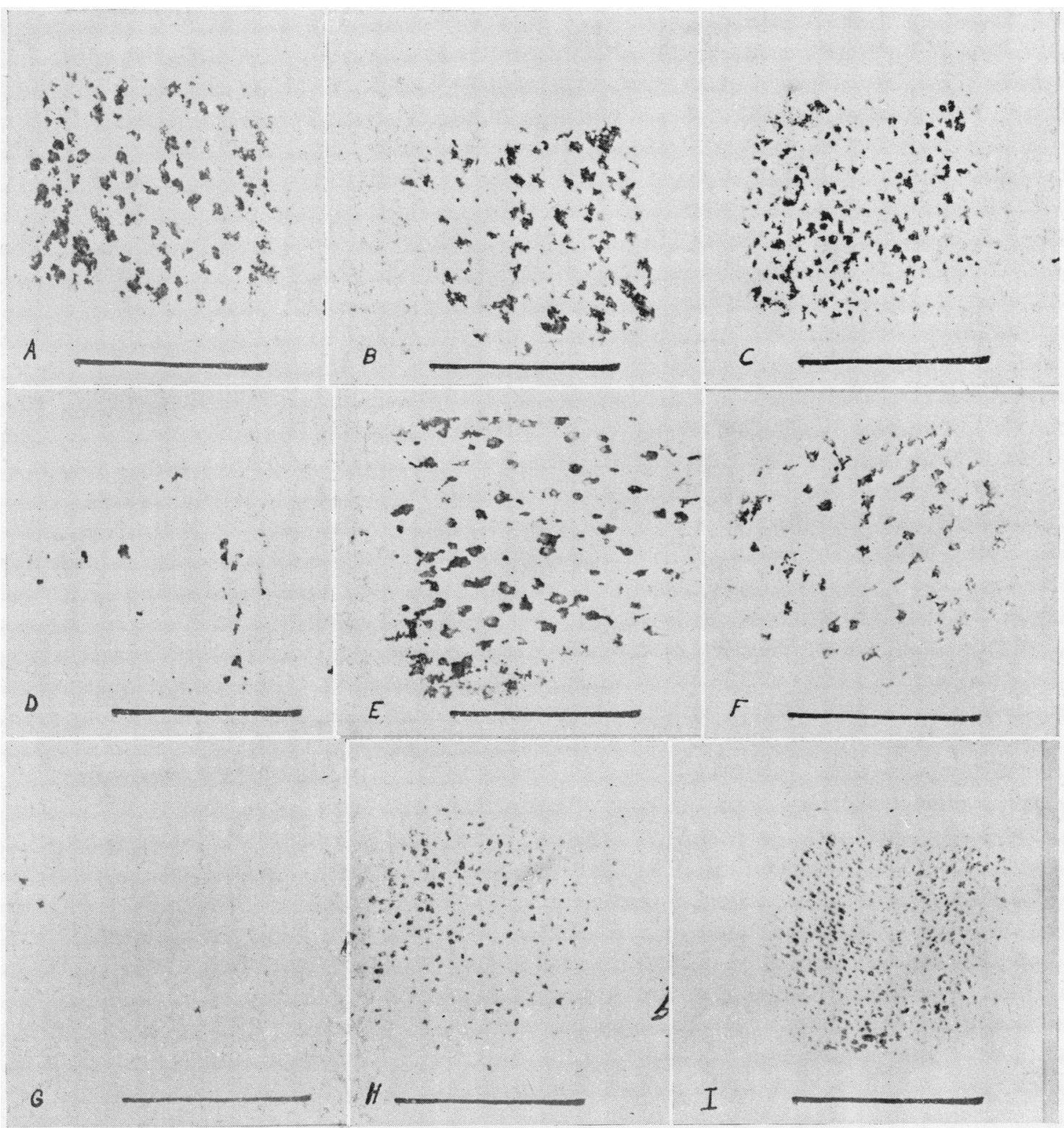

Fig. 3. Records of Maximum Number of Functional Sweat Glands Following Mecholyl Iontophoresis (Except in Segment I, Where No Drug Was Used) to Demonstate Patterns of Distribution on Different Body Surfaces

Calibration line equals $1 \mathrm{~cm}$. A. Medial surface of upper arm, over biceps. B. Extensor surface of forearm. C. Dorsum of hand. D. Scapular region of back. E. Anterior chest wall. F. Medial surface of leg over gastrocnemius. G. Buccal region of the face. H. Thenar eminence. I. Finger pad.

per gland hold particular significance in regulation of evaporative heat loss.

Cholinergic stimulation applied to different areas of the face revealed striking differences in the concentration of sweat glands. Administration of mecholyl into the skin of the zygomatic and buccal regions induced intense erythema and a local rise in skin temperature, but marked by very few functional sweat glands. This finding is in sharp contrast with temperature responses on areas in which mecholyl elicits a large blood flow accompanied by profuse sweating. Skin tempera- 
ture in such experiments does not increase markedly, and indeed, more often actually falls. It is a common experience, of course, in profuse sweating over the forehead region, to note the formation of large droplets which coalesce and run down over the zygomatic and buccal surfaces. In such situations, a high surface temperature in these areas might serve the useful purpose of increased evaporation, in spite of minimal local sweat output.

Somewhat inconstant results have been obtained following mecholyl iontophoresis on the forehead, as already described for the palmar surfaces. That is, occasionally we observe an actual depression of sweating following treatment. Such inconsistencies suggest the possibility of varying sweating responses of different areas to varying concentrations of the drug, or the possibility of functional differences in the innervation of these areas.

\section{COMMENT}

The method described has been used with considerable success in determining the extent of denervation in surgical removal of the sympathetic supply to the skin, and because of its simplicity has proved particularly adaptable to the hospital room. Simplicity of procedure is not attained at expense of precise and accurate detection however, as we have demonstrated many times. In 1 patient, over 200 sweat glands per sq. cm. were found to be functional as a result of mecholyl iontophoresis on the normal forearm, while only 125 glands were functional upon a similar area of the opposite arm 1 day after removal of a portion of the sympathetic supply. While the maximum number of functional glands remained constant on the normal arm, only 26 glands were evident 30 days after the operation, and only 6 remained functional 60 days after the operation. Evidently very few fibers supplying this area remained intact. Although degenerative processes may have been complete in the 2 or 3 weeks usually allowed, further loss of function continued for several weeks, probably as a result of fibrosis-producing blocks in the remaining intact pathways during the healing of the wound.

Similarly, precise detection of very slight, yet definite, sweating shown on the distal finger pads of the denervated hand in Figure 1 indicates the functional presence of relatively few intact fibers in the ulnar and median nerves, when compared with the 400 to 500 functional glands per sq. $\mathrm{cm}$. on the normal finger pads. Such precision in detection of intact nervous pathways has particular clinical significance in following the effects of peripheral sympathectomies, and in the important regenerative processes which follow.

The method has also been used in animal experiments involving monkeys, rats (9) and other laboratory animals. The animals need not be anesthetized, and only temporarily immobilized, therefore making information available under more nearly normal conditions.

\section{SUMMARY}

1. A method is described for the detection of sweating, and for enumeration of functional sweat glands in a given area under various kinds of stimulation. The method furnishes a quantitative measure of the number of active glands, and a qualitative measure of the amount of sweat secreted from each functional sweat pore. Its usefulness in checking the extent of denervation of sweating areas following sympathectomy is illustrated.

2. A comparison of thermal and direct cholinergic stimulation demonstrates that relatively severe thermal stimulation is required to activate as many glands as acetyl-beta-methylcholine (mecholyl). From theoretical considerations, the latter might be expected to activate the maximal number of functional glands in a given area in a given individual.

3. Maximum sweating consists of a combination of both increased numbers of participating sweat glands and increased output per gland, occurring in that order, with a demonstrable time interval separating the 2 levels of sweat output.

4. Patterns of distribution of sweat glands under maximum, or nearly maximum, stimulation indicate considerable numerical variation in sweat glands from area to area, and from individual to individual.

5. There is further evidence that sweat output per individual gland in different areas, and under different conditions, shows large variations.

\section{BIBLIOGRAPHY}

1. Minor, V., Eines Neues Verfahren zu der klinischen Untersuchung der Schweissabsonderung. Deutsche Ztschr. f. Nervenh., 1927, 101, 302. 
2. Roth, G. M., Clinical test for sweating. Proc. Staff Meet. Mayo Clin., 1935, 10, 383.

3. Silverman, J. J., and Powell, V. E., Simple technique for outlining sweat pattern. War Med., 1945, 7, 178.

4. Gurney, R., and Bunnell, I. L., Study of reflex mechanisms of sweating in the human being; effect of anesthesia and sympathectomy. J. Clin. Invest., 1942, 21, 269.

5. Kuno, Y., Variations in secretory activity of human sweat glands. Lancet, 1938, 1, 299.

6. Lobitz, W. C., Jr., and Osterberg, A. E., Chemistry of palmar sweat; preliminary report of apparatus and technic. J. Invest. Dermat., 1945, 6, 63.

7. Dale, H. H., and Feldberg, W., Chemical transmission of secretory impulses to sweat glands of cat. J. Physiol., 1934, 82, 121.

8. Kuno, Y., Significance of sweating in man. Lancet, 1930, 1, 912.

9. Ring, J. R., and Randall, W. C., Sweat glands in the rat and their response to prolonged nervous stimulation. Anat. Rec., 1946, 94, 34.

10. Buley, H. M., Active sweat glands, A method for their study. Arch. Dermat. and Syph., 1938, 38, 340. 